\title{
Selective mode excitation by nonaxial evanescent coupling for bandwidth enhancement of multimode fiber links
}

Laurent Vaissié

Eric G. Johnson

University of Central Florida

Find similar works at: https://stars.library.ucf.edu/facultybib2000 University of Central Florida Libraries http://library.ucf.edu

This Article is brought to you for free and open access by the Faculty Bibliography at STARS. It has been accepted for inclusion in Faculty Bibliography 2000 s by an authorized administrator of STARS. For more information, please contactSTARS@ucf.edu.

\section{Recommended Citation}

Vaissié, Laurent and Johnson, Eric G., "Selective mode excitation by nonaxial evanescent coupling for bandwidth enhancement of multimode fiber links" (2002). Faculty Bibliography 2000s. 3516.

https://stars.library.ucf.edu/facultybib2000/3516

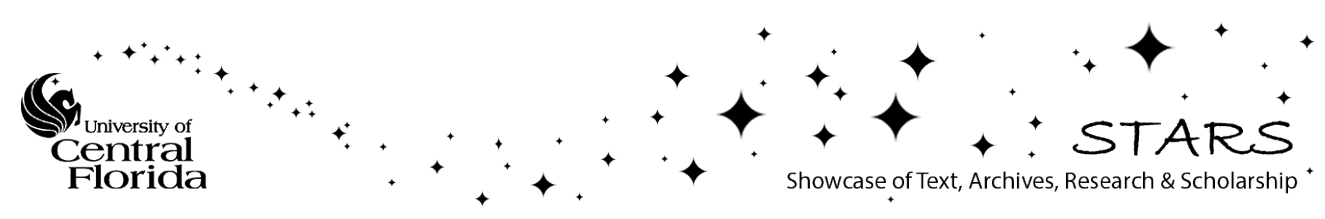




\section{Selective mode excitation by nonaxial evanescent coupling for bandwidth enhancement of multimode fiber links}

\author{
Laurent Vaissié \\ Eric G. Johnson \\ University of Central Florida \\ CREOL/School of Optics \\ 4000 Central Florida Boulevard \\ Orlando, Florida 32816-2700 \\ E-mail: Ivaissie@mail.ucf.edu
}

\begin{abstract}
We present an analysis of conditioned launch in a gradientindex multimode fiber (MMF). Selective mode excitation is obtained by nonaxial evanescent coupling between two highly asymmetric sidepolished fibers: a single-mode fiber and an MMF with an exposed core. An index-matching slab improves the coupling efficiency and selectivity. The simulation shows that about $40 \%$ of modes, only high-order modes, are excited in the MMF. When compared to an overfilled launch, the bandwidth of a perturbed index fiber is enhanced more than twofold for large parameter variations. () 2002 Society of Photo-Optical Instrumentation Engineers. [DOI: 10.1117/1.1488607]
\end{abstract}

Subject terms: local area networks; optical fibers; evanescent coupling; gradient index.

Paper 010289 received Aug. 15, 2001; revised manuscript received Feb. 11, 2002; accepted for publication Feb. 13, 2002.

\section{Introduction}

The computer interconnects in local area networks (LANs) are today required to transmit data at very high rates to satisfy the increasing bandwidth demand. The IEEE 802.3 committee underwent a great effort in the past few years to achieve and standardize a rate of gigabit per second data transmission for these local networks. ${ }^{1}$ The standardization approved by the committee includes data transmission by the already installed gradient-index multimode fibers (MMFs) that constitute the dominant backbone of these networks. These MMFs are operated at 850 or at $1300 \mathrm{~nm}$ and the minimum bandwidth requirement for a longwavelength 1300-nm link (LX) was fixed to be $500 \mathrm{MHz}$ $\mathrm{km}$ for a $62.5-\mu \mathrm{m}$ core fiber. ${ }^{1}$

The first technique used to launch light and excite a maximum number of modes in the MMF consists of radially overfilling the MMF with a light-emitting diode onaxis. Because the source is spatially incoherent, almost all the modes in the MMF are excited with the same amplitude factor. This technique is called overfilled launch (OFL). This launching method would be optimal if the actual fiber index of refraction matched the theoretical dispersion-free profile that is close to parabolic.

However, defects in the gradient-index profile of installed gradient index fibers due to the modified chemical vapor deposition (MCVD) fabrication process limit the maximum bandwidth achievable by the OFL method, as first pointed out by Checcacci et al. ${ }^{2}$ and investigated numerically by Marcuse. ${ }^{3}$ Index variations such as central depression and core-cladding interface dip cause the group velocity to vary from one mode to another. Consequently each mode will propagate in the fiber at a slightly different velocity with respect to each other, causing the pulse to stretch as it travels from transmitter to receiver. The impulse response of the fiber is therefore stretched and the corresponding bandwidth, defined as the Fourier transform of the impulse response, is limited. The resulting dispersion of index profile defect is referred to as differential mode delay (DMD) and is generally worse if all the modes are excited. The dispersion is more important for trajectories where the index differs significantly from the perfect parabolic case, in such locations as the axis or the core-cladding interface.

The measured bandwidth of such fibers was found to be far below the $500 \mathrm{MHz} \mathrm{km}$ requirement of the IEEE standard for a $62.5-\mu \mathrm{m}$ core fiber at $1300 \mathrm{~nm}$. The general solution to the DMD problem is therefore to excite only a few modes in the MMF. The idea is to limit the intermodal dispersion and therefore restore available bandwidth by exciting only modes propagating along trajectories where the refraction index matches the dispersion-free profile.

Several methods have been investigated for selective mode excitation in a gradient-index fiber. One method consists of exciting high-order modes of the MMF through butt-coupling with a single-mode fiber (SMF) off axis. This method was first presented by Jeunhomme and Pocholle ${ }^{4}$ for index profile measurement and applied to bandwidth enhancement by Webster et al., ${ }^{5}$ Raddatz et al., ${ }^{6,7}$ and Johnson and Stack. ${ }^{8}$ It was shown that this method enables us to achieve a bandwidth comparable to the OFL.

Another method investigated uses a vortex lens to phase match the launched field to a couple of modes by transforming a plane wave to a donut-shaped energy distribution with a spiral phase to couple light to a very restricted number of modes. ${ }^{8}$ However, it requires a diffractive element to transform the launched field into a donut shape.

Webster et al. pointed out that launching light at an angle greater or equal to 6 deg could prevent bandwidth collapse in MMF links. ${ }^{5}$ Following this analysis, the method presented here employs tilted evanescent coupling from a side-polished single-mode (SPSM) to a side- 
polished gradient-index fiber (SPMM) to launch skew rays and excite higher order modes. Bandwidth enhancement ratios at least similar to the offset launch technique are expected using this technique.

First, we will describe the numerical framework of this paper. We consider an index profile close to parabolic case but perturbed by a central depression and a core-cladding negative distortion. Rigorous ray tracing in the gradientindex fiber is calculated and compared to energy trajectories of excited modes simulated by the beam-propagation method. The ray simulation is used to confirm that the coupling technique excites modes propagating along defectfree paths of the index profile.

From the perturbed profile we derive the DMD that is scaled to the $2 \mathrm{~ns} / \mathrm{km}$ limit set by the gigabit Ethernet committee. The bandwidth is then computed consistently with the procedure described by Marcuse and used previously in the literature. ${ }^{7}$

In the second part of the paper, we present the results of bandwidth enhancement over the OFL bandwidth for the new method based on evanescent coupling. The effective number of excited modes is also presented. We then discuss the results to determine which method should be used in LANs.

\section{Nonaxial Evanescent Coupling of Side-Polished Fibers}

The key to achieve bandwidth enhancement of perturbedindex profile MMFs is to excite a limited number of modes that will propagate along trajectories where the index does not present large deviation. To do so, we propose to use the technique of evanescent coupling between an SPSM and an SPMM. The mechanical polishing technique is well known now and fabrication of such elements is described in numerous papers. ${ }^{9}$ The control of polishing depth may be controlled to within an accuracy ${ }^{10}$ of $\pm 0.25 \mu \mathrm{m}$.

Coupling between asymmetric fibers has been investigated for more than a decade, including adiabatic and sidepolished couplers. ${ }^{11}$ They have been proven useful as a flexible alternative to a $3-\mathrm{dB}$ coupler in a linear data bus ${ }^{12}$ or a nonreciprocal directional coupler. ${ }^{13}$ Valentin and Igor also investigated oblique coupling between two dissimilar fibers through fused tapered fibers. Their concept was recently applied to pumping of multimode fibers. ${ }^{14}$

In this paper, we propose to investigate a nonaxial evanescent coupling scheme between highly asymmetric fibers and its application to bandwidth enhancement of nonperfect MMFs in LANs. We also investigate the role of an intermediate index-matching slab between the fibers for improved coupling efficiency and selectivity.

Figure 1 represents the coupling scheme proposed for evanescent coupling. An SMF is polished down to within 2 $\mu \mathrm{m}$ close to the core. This fiber is used to launch light in a gradient index fiber that was polished until the core was exposed. The distance between the axis of the MMF and the cladding plane is the polishing depth. The MMF presents an exposed core for two reasons. First, the coupling will have a better bandwidth enhancement ratio if the light is not launched at the core-cladding discontinuity. Second, the coupling will be more efficient if the index of refraction in the core of the SMF and at the cladding plane of the MMF are similar. A 3- $\mu$ m-thick layer of index matching

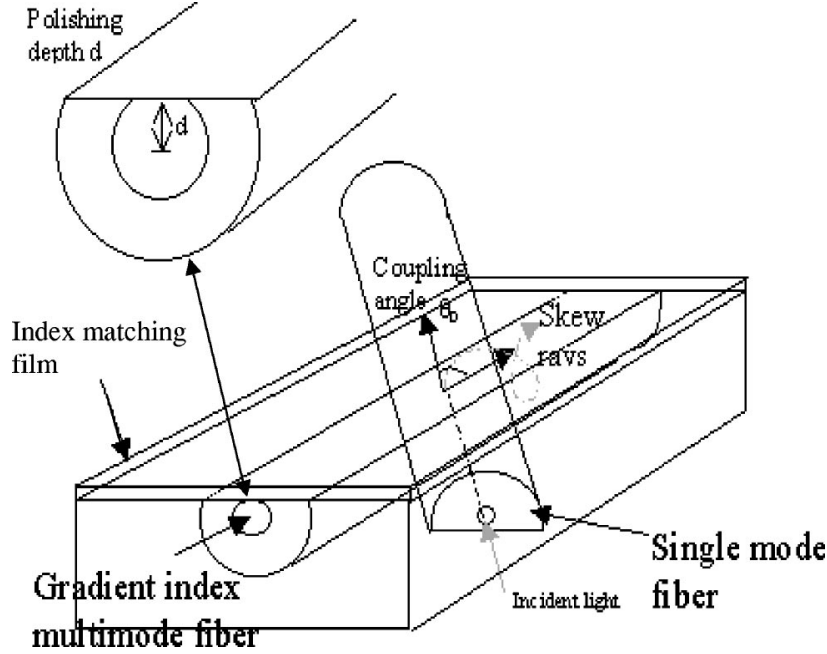

Fig. 1 Proposed evanescent coupling scheme between SPSM and SPMM. The MMF presents a perturbed parabolic profile and is polished to a distance $d$ from the axis. The SMF cladding plane is at 2 $\mu \mathrm{m}$ above the core. The bandwidth enhancement is investigated with respect to the coupling angle $\theta_{0}$ and the polishing depth $d$ of the MMF.

fluid ( $n=1.457$ ) is used to enhance the coupling efficiency, which could also be achieved by depositing an oxide film on the SPMM. Its important role for coupling efficiency is demonstrated in Sec. 4.3.

The index profile of the MMF presents an important central depression on axis, where DMD was shown to increase dramatically. ${ }^{2,7}$ Thus we propose to launch light into skew rays of the SPMM by coupling at an angle between the two side-polished fibers. For the simulations the angle varies from 2 to $10 \mathrm{deg}$.

\section{Framework of Investigation}

\subsection{Perturbed Index Profile}

The MCVD fabrication process mainly yields defects at two distinct locations in a gradient-index MMF, on axis and at the core cladding interface. ${ }^{4}$ These perturbed profiles have been investigated in different DMD surveys and a statistical investigation of a profile variation based on these perturbations helped to provide some insight into the possible bandwidth enhancement obtained with an offset launch technique. Based on these studies, we consider in this paper a case combining a central depression and discontinuity of refractive index distribution at the corecladding interface. The index of refraction in the core falls abruptly to equal the cladding index at a radius of $28 \mu \mathrm{m}$, considering a $62.5-\mu \mathrm{m}$ core fiber. The central depression is modeled by a 5 - $\mu$ m-radius central dip such that $\Delta n$ $=n(5)-n(0)=0.0044$. The cladding index is 1.457 .

The departure of the index profile with respect to the perfect parabolic profile $n=1.477[1-2 \times 0.0135$ $\left.\times(r / 31)^{2}\right]^{1 / 2}$ is shown in Fig. 2 .

The next section describes the derivation of ray equations for a parabolic index profile MMF. These equations are used in comparison with the beam propagation results to confirm that light is launched along helical paths. 


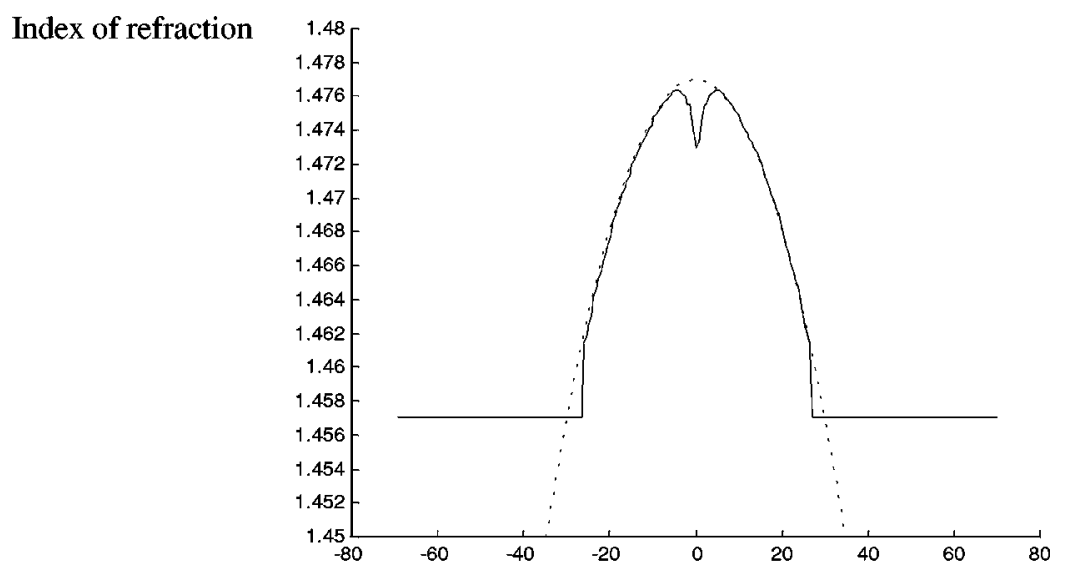

\section{Radial coordinate (microns)}

Fig. 2 Perturbed index profile of the MMF and perfect parabolic profile (dashed line). The perturbed index presents a $5-\mu \mathrm{m}$ wide central depression and a core-cladding discontinuity located $3 \mu \mathrm{m}$ before the cladding.

\subsection{Ray Equations in Parabolic Index Multimode Fiber}

Following the framework of Cozannet et al., ${ }^{15}$ we derive the equations describing the propagation of rays in optical fibers with parabolic gradient-index without approximations. The ray parameters are defined in Fig. 3. In cylindrical coordinates the vector $\mathbf{O M}$ can be expressed as

$\mathbf{O M}=\mathbf{r}=\rho \mathbf{u}_{r}+z \mathbf{k}$.

And the tangent vector $\mathbf{T}$ is defined by

$$
\begin{aligned}
\mathbf{T}=\frac{\mathrm{d} \mathbf{r}}{\mathrm{d} s} & =\frac{\mathrm{d} \rho}{\mathrm{d} s} \mathbf{u}_{r}+\rho \frac{\mathrm{d} \mathbf{u}_{r}}{\mathrm{~d} s}+\frac{\mathrm{d} z}{\mathrm{~d} s} \mathbf{k} \\
& =\sin (\theta) \cos (\varphi) \mathbf{u}_{r}+\sin (\theta) \sin (\varphi) \mathbf{u}_{\varphi}+\cos (\theta) \mathbf{k}
\end{aligned}
$$

We assume the index is dependent only on $\rho$ as we investigate gradient-index fibers.

The ray equation can be written as

$$
n \frac{\mathrm{d} \mathbf{r}}{\mathrm{d} s}=\operatorname{grad}(S)
$$

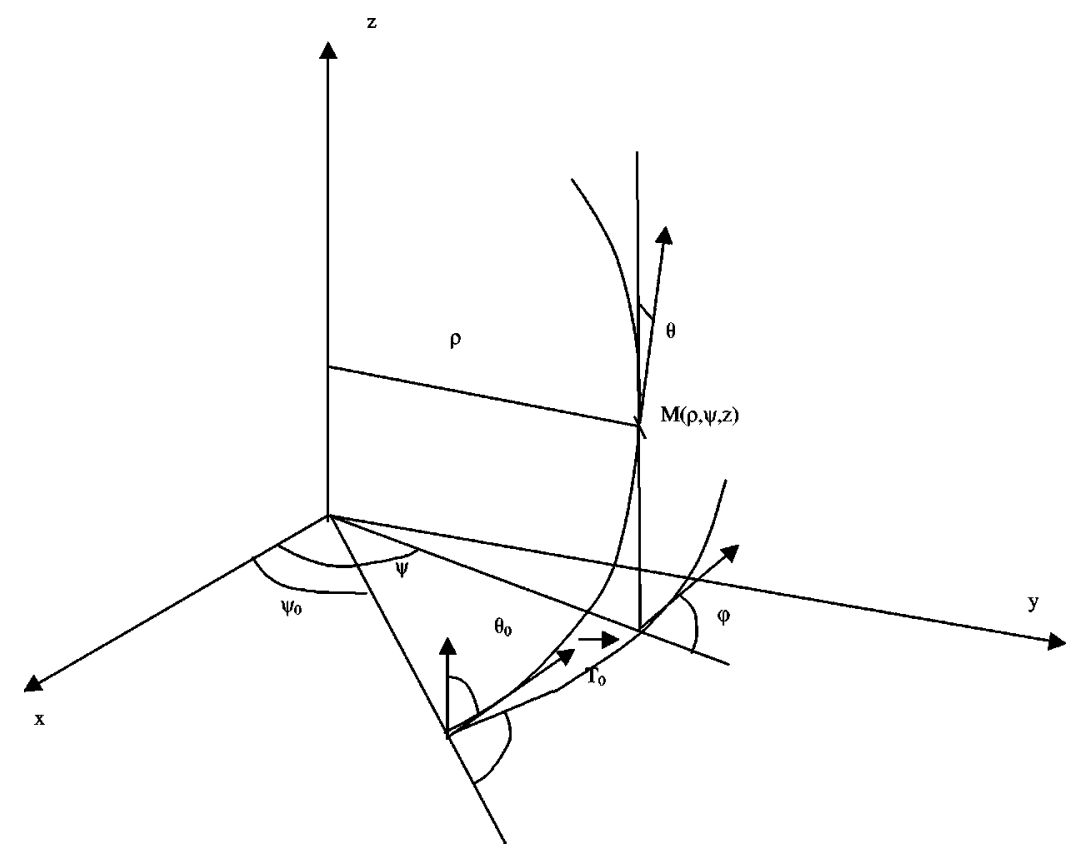

Fig. 3 Ray parameters. The $z$ axis defines the axis of the multimode fiber. 
where $S$ is a surface of constant phase. Taking the derivative with respect to $s$ leads to ${ }^{1}$

$\frac{\mathrm{d}}{\mathrm{d} s}\left(n \frac{\mathrm{d} \mathbf{r}}{\mathrm{d} s}\right)=\operatorname{grad}(n)$.

By projection on $\left(\mathbf{u}_{r}, \mathbf{u}_{\varphi}, \mathbf{k}\right)$ and using

$\frac{\mathrm{d} \mathbf{u}_{r}}{\mathrm{~d} s}=\frac{\mathrm{d} \psi}{\mathrm{d} s} \mathbf{u} \varphi$

$\frac{\mathrm{d} \mathbf{u}_{\varphi}}{\mathrm{d} s}=-\frac{\mathrm{d} \psi}{\mathrm{d} s} \mathbf{u}_{r}$

Eq. (4) becomes the system ${ }^{15}$

$n\left(\frac{\mathrm{d} \psi}{\mathrm{d} s}\right)^{2}\left(\rho^{\prime \prime}-2 \frac{\rho^{\prime 2}}{\rho}-\rho\right)=\frac{\mathrm{d} n}{\mathrm{~d} \rho}$,

$n\left(\frac{\mathrm{d} \psi}{\mathrm{d} s}\right) \rho^{2}=\alpha=n\left(\rho_{0}\right) \rho_{0} \sin \left(\theta_{0}\right) \sin \left(\varphi_{0}\right)$,

$n\left(\frac{\mathrm{d} \psi}{\mathrm{d} s}\right) z^{\prime}=n\left(\rho_{0}\right) \cos \left(\theta_{0}\right)$

where the derivative with respect to $\psi$ is designed by a prime.

Using the new variable $v=1 / \rho$, Eqs. (5) and (6) give

$\alpha^{2}\left(v^{\prime \prime}+v\right)=n\left(\frac{\mathrm{d} n}{\mathrm{~d} v}\right)$

We consider now the parabolic index profile given by

$n=n_{1}\left(1-2 \Delta\left(\frac{\rho}{a}\right)^{2}\right)^{1 / 2}$ if $\rho \leqslant a$

$n=n_{2} \quad$ if $\rho>a$,

where $\Delta=\left(n_{1}^{2}-n_{2}^{2}\right) /\left(2 n_{1}^{2}\right)$ and $a$ is the core radius of fiber.

Then Eq. (8) becomes

$v^{2}\left(v v^{\prime \prime}+v^{2}\right)=\frac{2 n_{1}^{2} \Delta}{\alpha^{2} a^{2}}=\gamma^{2}$,

The general solution of Eq. (9) is

$v^{2}=A \cos \left[2\left(\psi-\psi_{i}\right)\right]+\left(A^{2}+\gamma^{2}\right)^{1 / 2}$.

Equation (10) defines an ellipse where the great axis $\rho_{M}$ and small axis $\rho_{m}$ are obtained for $\psi=\psi_{i}$ and $\psi=\psi_{i}$ $+\pi / 2$, respectively.

$\rho_{m}=\left[\frac{1}{-A+\left(A^{2}+\gamma^{2}\right)^{1 / 2}}\right]^{1 / 2}$,

$\rho_{M}=\left[\frac{1}{A+\left(A^{2}+\gamma^{2}\right)^{1 / 2}}\right]^{1 / 2}$.
The initial conditions are defined by $\mathbf{O M}_{0}\left(\rho_{0}, 0, z_{0}\right)$ and $\mathbf{T}_{0}$ $\left[\sin \left(\theta_{0}\right) \cos \left(\phi_{0}\right), \sin \left(\theta_{0}\right) \sin \left(\phi_{0}\right), \cos \left(\theta_{0}\right)\right]$. The constants $A$ and $\psi_{i}$ are obtained by rewriting Eq. (2) such that

$$
\begin{aligned}
\mathbf{T} & =\left(\frac{\mathrm{d} \psi}{\mathrm{d} s}\right) \frac{\mathrm{d} \rho}{\mathrm{d} \psi} \mathbf{u}_{r}+\rho \frac{\mathrm{d} \psi}{\mathrm{d} s} \mathbf{u} \varphi+\frac{\mathrm{d} z}{\mathrm{~d} s} \mathbf{k} \\
& =\sin (\theta) \cos (\varphi) \mathbf{u}_{r}+\sin (\theta)(\sin )(\varphi) \mathbf{u}_{\varphi}+\cos (\theta) \mathbf{k}
\end{aligned}
$$

From which one can derive

$\tan (\varphi)=\frac{\rho}{\rho^{\prime}}=\frac{1}{A \rho^{2} \sin \left[2\left(\psi-\psi_{i}\right)\right]}$

Plugging the result into Eq. (10) yields

$A=\frac{\left\{1+\left[2 \cos \left(\varphi_{0}\right)\right] /\left[\sin \left(\varphi_{0}\right)\left(1-\rho_{0}^{4} \gamma^{2}\right)\right]\right\}^{1 / 2}\left(1-\rho_{0}^{4} \gamma^{2}\right)}{2 \rho_{0}^{2}}$,

$\psi_{i}=\psi_{0}+\frac{\arctan \left\{2 /\left[\tan \varphi_{0}\left(1-\rho_{0}^{4} \gamma^{2}\right)\right]\right\}}{2}$.

Then one can express $\rho$ as a function of $z$ by integrating Eq. (7) to eliminate $\psi$, which yields

$\rho^{2}=\rho_{M}^{2}\left\{\sin ^{2}\left[\frac{2 \pi}{T}\left(z-z_{i}\right)\right]+\left(\frac{\rho_{m}}{\rho_{M}}\right)^{2} \cos ^{2}\left[\frac{2 \pi}{T}\left(z-z_{i}\right)\right]\right\}$,

where

$$
\begin{aligned}
T & =\frac{2 \pi n\left(\rho_{0}\right) \cos \theta_{0} a}{n_{1} \sqrt{2 \Delta}} \\
z_{i} & =z_{0}+\frac{T}{2 \pi} \arctan \left[\frac{\rho_{m}}{\rho_{M}} \tan \left(\psi_{i}\right)\right] .
\end{aligned}
$$

Taking into account the rotation of the axis of the ellipse with respect to $(x, y)$ set of axis, the ray coordinates are then defined in Cartesian coordinates by

$$
\left(\begin{array}{l}
x \\
y
\end{array}\right)=\left\{\begin{array}{l}
\rho_{m} \cos \left[\frac{2 \pi}{T}\left(z-z_{i}\right)\right]^{*} \cos \left(\psi_{i}\right)-\rho_{M} \sin \left[\frac{2 \pi}{T}\left(z-z_{i}\right)\right]^{*} \sin \left(\psi_{i}\right) \\
\rho_{m} \cos \left[\frac{2 \pi}{T}\left(z-z_{i}\right)\right]^{*} \sin \left(\psi_{i}\right)+\rho_{M} \sin \left[\frac{2 \pi}{T}\left(z-z_{i}\right)\right]^{*} \cos \left(\psi_{i}\right)
\end{array}\right\} .
$$

\subsection{Modal Fields and Propagation Constants of Perturbed-Index Profile MMF}

Given the index profile presented in Fig. 2, we used the mode solver module of beam propagation method (BPM)$\mathrm{CAD}$ to find its propagating mode fields as well as the corresponding propagation constants. Given a 2-D permittivity distribution, the algorithm assumes the waveguide is $z$-invariant and launches a Gaussian input field off axis for imaginary propagation. The alternating direction implicit method, which splits the propagation operator along the $x$ 
and $y$ directions, is applied to scalar Helmholtz equation. ${ }^{16}$ To compute the propagation constants, the imaginary distance beam propagation method is used. This approach considers the input field as sum of propagating modes and compares their gain after imaginary propagation steps along the $z$ axis. ${ }^{17}$ Since the fundamental mode has the largest effective mode index, it will see the maximum gain after a large enough propagation step. The effective mode index $n_{\text {eig }}$ is then obtained by considering steady state growth rate of the mode following the equation

$$
\begin{aligned}
k n_{\text {eig }}= & k n_{0}+\lim _{z \rightarrow \infty} \\
& \times \frac{\ln \left[\int E(x, y, z+\Delta z) \mathrm{d} x \mathrm{~d} y\right]-\ln \left[\int E(x, y, z) \mathrm{d} x \mathrm{~d} y\right]}{\Delta z} .
\end{aligned}
$$

To find higher order modes, the fundamental mode is subtracted from the field obtained after a propagation step and the procedure is reiterated.

We ran the simulation over a $502 \times 502$ regular mesh grid covering a $70 \times 70-\mu \mathrm{m}^{2}$ area centered on the gradient index fiber. A mode field and effective index are found when they differ by less than the convergence factor after one propagation step. The convergence tolerance factor was set to $1 \times 10^{-7}$ for the modal effective index and $1 \times 10^{-5}$ for the field amplitude. The program found 50 scalar modes propagating in the fiber at $1300 \mathrm{~nm}$.

Another simulation was run at $1310 \mathrm{~nm}$ to derive the transit time per unit length $L$ for each mode given by

$\left|\tau_{i}\right|=\frac{1}{L}\left|\frac{\mathrm{d} \beta}{\mathrm{d} \omega}\right| \approx\left|\frac{\Delta \beta}{\Delta \omega}\right| \approx \frac{\Delta \beta \lambda^{2}}{2 \pi c \Delta \lambda}$,

with $\Delta \lambda=10 \mathrm{~nm}, c=3 \times 10^{8} \mathrm{~m} / \mathrm{s}, \lambda=1300 \mathrm{~nm}$. For each mode, one can then derive the DMD as a function of mode order.

\subsection{Impulse Response and Bandwidth}

The impulse response can then be computed for a specific input field by attributing a coefficient $C_{i}$ to each mode corresponding to the overlap integral of the mode field with the input field such that

$C_{i}=\varepsilon_{i} \frac{\left|\iint_{x y} E_{L} E_{i}^{\prime} \mathrm{d} x \mathrm{~d} y\right|^{2}}{\iint_{x y}\left|E_{L}\right|^{2} \mathrm{~d} x \mathrm{~d} y \iint_{x y}\left|E_{i}\right|^{2} \mathrm{~d} x \mathrm{~d} y}$,

with

$\varepsilon_{i}=2$ if $i=0,1$

$\varepsilon_{i}=4$ if $i \neq 0,1$.

The coefficient $\varepsilon_{i}$ takes into account the degeneracy of polarization and helical direction of propagation of the $i$ 'th mode. These coefficients are referred to as scaled power overlap integral coefficients. A BPM propagation of a few

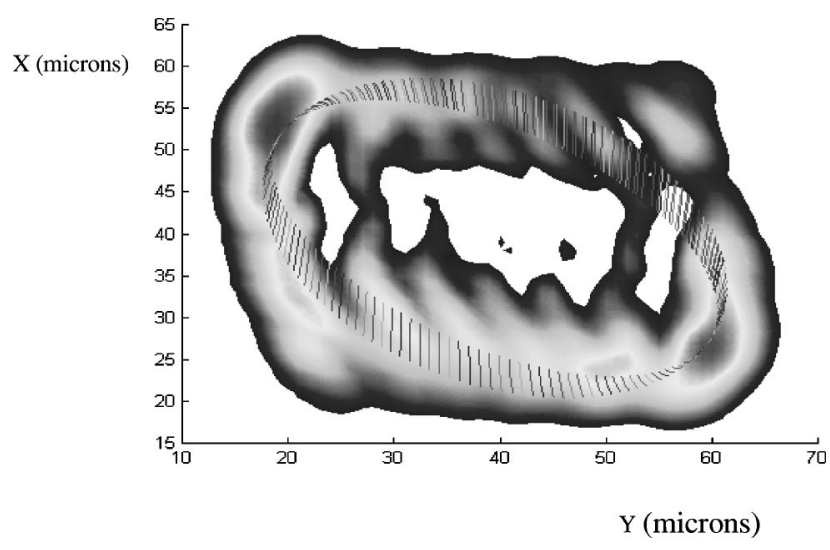

Fig. 4 Energy distribution in the core of the MMF after coupling with parameters $d=18 \mu \mathrm{m}$ and $\theta_{0}=4 \mathrm{deg}$. The field intensity was summed over a $1000-\mu \mathrm{m}$ propagation. The intensity shows a welldefined donut shaped. The ray trajectories launched with the coupling parameters and taking the divergence of the beam into account are projected on the figure (dashed lines).

millimeters is used to propagate the field in the gradientindex fiber and eliminate the modes with high losses before computing the overlap integral.

The coefficients $C_{i}$ of the modes, for which DMD falls into an interval $\Delta \tau$, are then summed to obtain a staircase approximation of the impulse response. This method, described first by Marcuse, eliminates the artifacts in bandwidth derivation due to a smoothing of the impulse response. $^{3}$ The interval $\Delta \tau$ is generally set to

$\Delta \tau=\frac{\tau_{\max }-\tau_{\min }}{n_{\max }}$

where $n_{\max }$ is a power of two for use of fast Fourier transform (FFT). Noted that $\Delta \tau$ is adjusted for each case to avoid aliasing of the impulse response.

When deriving the OFL bandwidth one assumes all the modes are excited equally and therefore

$C_{i}=1, \quad \forall i$

Although this is practically never the case with any launching condition, this assumption generally enables one to get close to the worst-case bandwidth. The bandwidth derived in our case was found to be well below the 500 MHz commonly admitted to be the "worst case" one could find with legacy fibers. Thus the index profile we investigate belongs to the 38 fibers group out of 81 studied by Webster et al., ${ }^{5}$ which present a DMD larger than $2 \mathrm{~ns} / \mathrm{km}$. The bandwidth is then derived as the full width half maximum of the FFT of the impulse response, which represents the $3-\mathrm{dB}$ point of the optical power.

\section{Simulation Results}

\subsection{Beam Propagation Simulation}

We simulated the coupling using a scalar beam propagation method (BPM-CAD 4.0, Optiwave Corporation, Canada 1999). Figure 4 shows a $X Y$ projection of the field propagated in the multimode fiber over a period of rays trajectory 


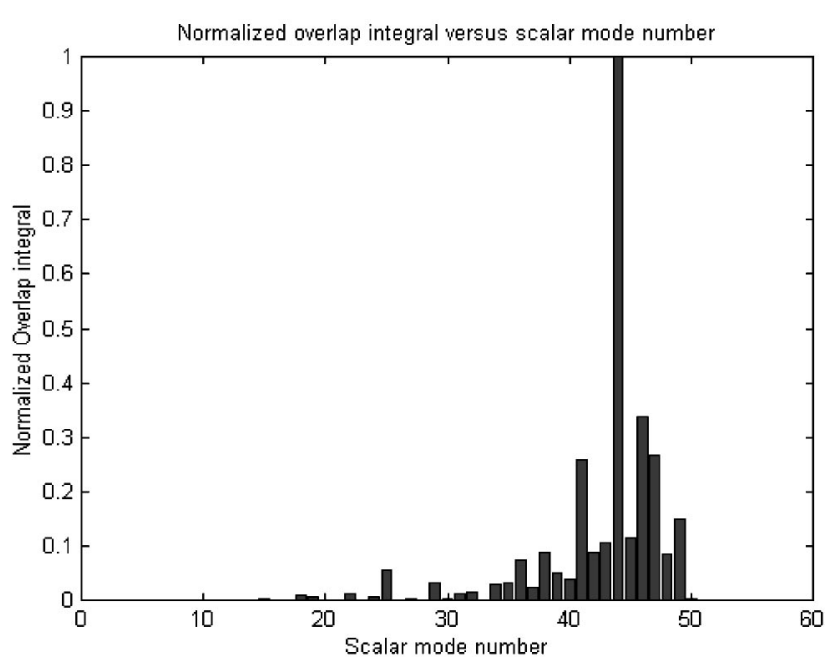

Fig. 5 Normalized overlap integral of propagating field and degenerate LP modes with respect to scalar mode number. Parameters are $d=16 \mu \mathrm{m}$ and $\theta_{0}=8 \mathrm{deg}$.

as derived in Sec. 3.2. This figure illustrates the donutshaped energy distribution in the core of the MMF. Almost no power is found on axis. This property limits the influence of index central depression and therefore enhances the bandwidth.

We superimposed the ray trajectories calculated for a perfect parabolic profile. The analytical equations are presented in Sec. 3.2. The rays are launched with the parameters pertaining to the coupling and present a good fit to energy trajectories, reinforcing the evidence that the light is propagating along skew rays.

\subsection{Overlap Integral}

The scalar field obtained in the core of the SPMM by beam propagation simulation of evanescent coupling is then compared to the degenerate LP mode electric fields that may be excited in this fiber. The overlap integral as defined by Eq. (24) is shown in Fig. 5 for a coupling angle of 8 deg and a polishing depth of $16 \mu \mathrm{m}$. This figure illustrates the selec- tive excitation of high-order modes by the evanescent coupling technique. The effective number of modes excited in the MMF is given, following Raddatz, by

$m_{\mathrm{eff}}=\frac{\left(\sum_{i} C_{i}\right)^{2}}{\sum_{i} C_{i}^{2}}$.

The effective mode number $m_{\text {eff }}$ is plotted as a function of coupling angle and polishing depth in Fig. 6. The variation of $m_{\text {eff }}$ shows the number of excited modes decreases as the offset increases. The simulation shows $50 \%$ of modes are excited for a polishing depth of $10 \mu \mathrm{m}$ at a 6-deg coupling angle but only $20 \%$ are excited at a distance of 18 $\mu \mathrm{m}$. A coupling angle around $6 \mathrm{deg}$ appears to be the best choice to excite a maximum of modes while improving bandwidth and making the technique insensitive to mode mixing incurred during propagation.

As a comparison, we used the method described here to evaluate the offset launch technique. We simulated the propagation of light launched from a 8 - $\mu \mathrm{m}$-diameter SMF butt-coupled to the gradient-index fiber for different offsets. The effective mode number is plotted in Fig. 7 as a function of the offset. A maximum effective number of 12 modes appear to be excited by the offset technique. Thus, the evanescent coupling method excites twice as many modes. We must, however, acknowledge that this number will increase if the SMF is placed farther away from the MMF since the diffraction of the Gaussian beam will yield a larger waist diameter that will be likely to excite more modes.

\subsection{Bandwidth Enhancement Ratio}

We define the bandwidth as 3-dB points of the FFT of the impulse response. The impulse response was stair-cased, as described in Marcuse. ${ }^{3}$ The bandwidth obtained by evanescent coupling technique was then divided by the bandwidth obtained for OFL technique where all the degenerate LP modes are equally excited. The results are shown in Fig. 8. The enhancement ratio appears to be greater than two for a large variation of polishing depth parameters $( \pm 4 \mu \mathrm{m})$ and coupling angle $( \pm 4 \mathrm{deg})$. The enhancement ratio increases

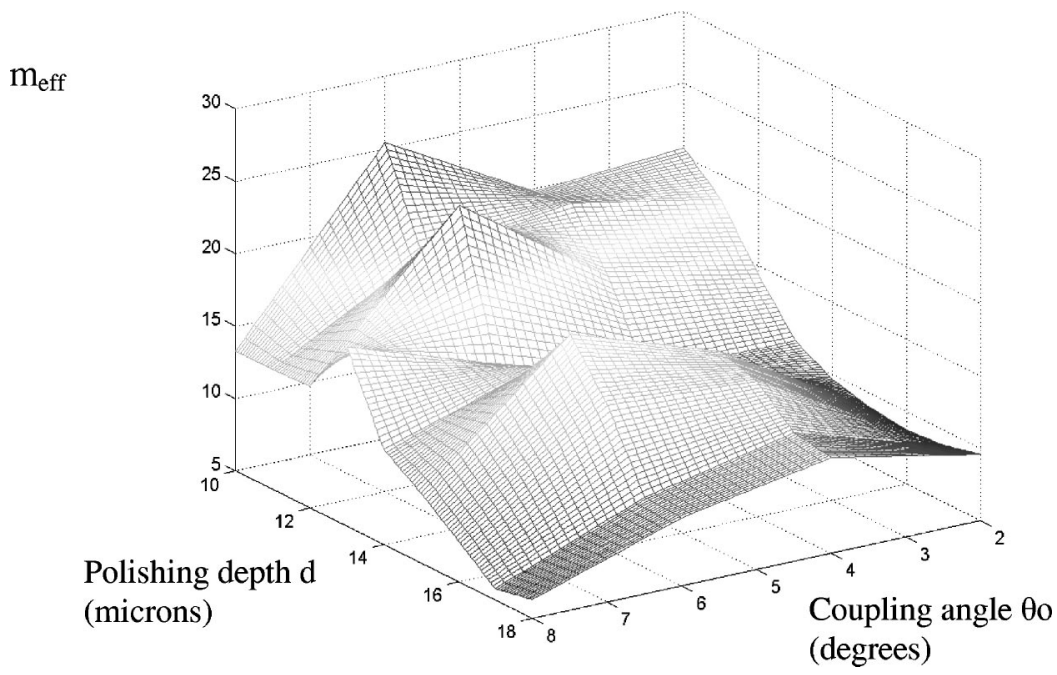

Fig. 6 Effective number of excited modes in the MMF after coupling out of 50 degenerate LP modes. 


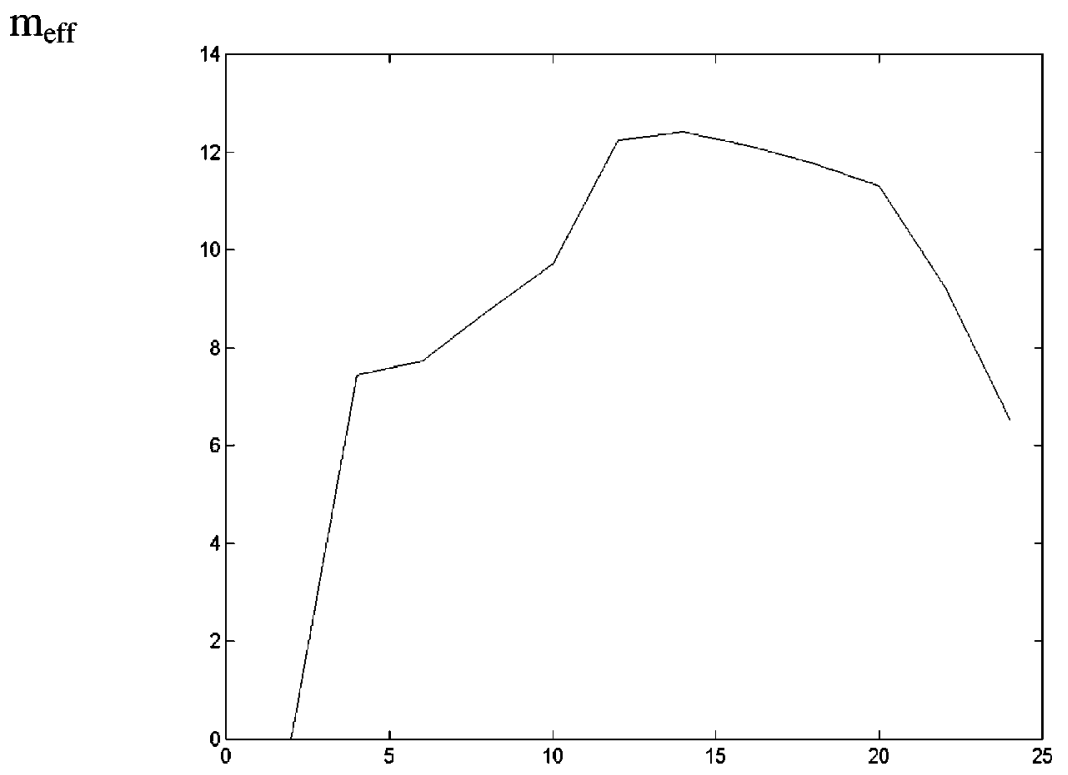

\section{Offset (microns)}

Fig. 7 Effective number of excited modes obtained with offset launch technique as a function of the offset. The maximum number of propagating modes is 50 .

as the coupling angle increases, showing the importance of the coupling angle for a fixed offset. As the polishing depth increases, more modes are excited close to the core cladding interface and the ratio decreases. The maximum enhancement ratio is obtained for polishing depth of $18 \mu \mathrm{m}$ and coupling angle of $6 \mathrm{deg}$.

The bandwidth enhancement obtained when simulating the classic offset technique showed to be limited to a factor of 2 for any offset and no tilt angle.

\subsection{Role of Index Matching Slab in Coupling Efficiency and Selectivity}

We investigated the coupling efficiency of the launch technique using the BPM. Light was launched as the fundamental mode of the SMF. The power coupled into the MMF

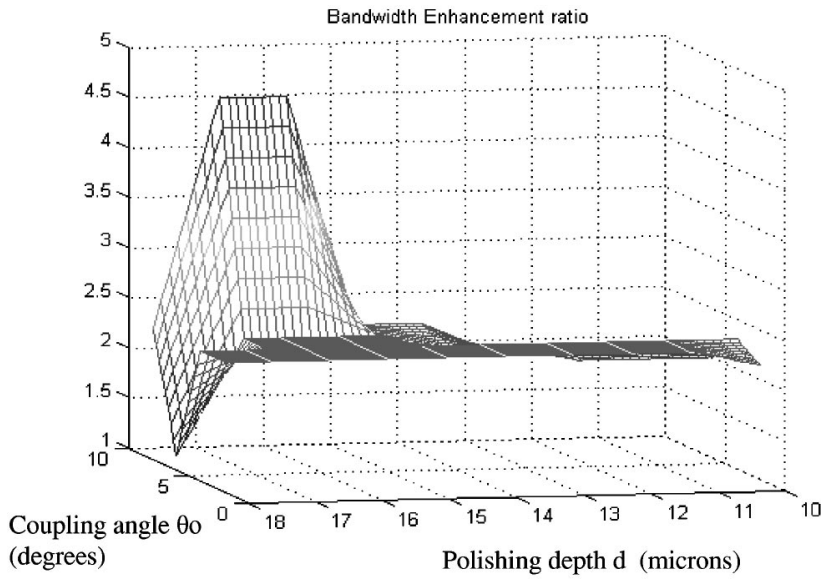

Fig. 8 Bandwidth enhancement ratio with respect to bandwidth obtained by OFL as a function of polishing depth and coupling angle. was computed after a $2.1 \mathrm{~mm}$ propagation distance. At this distance, there is no longer any interaction between the waveguides. The simulation was run for angles of 2,4 , and 6 deg between the axis of the fibers as well as for several polishing depths. A comparison between coupling efficiency with and without a slab is presented in Fig. 9.

The results show the importance of the index-matching slab for nonaxial coupling. Without the slab, the coupling efficiency drops dramatically for angles greater than $2 \mathrm{deg}$ to levels that make the technique useless. On the other hand, the presence of the slab enhances the coupling and

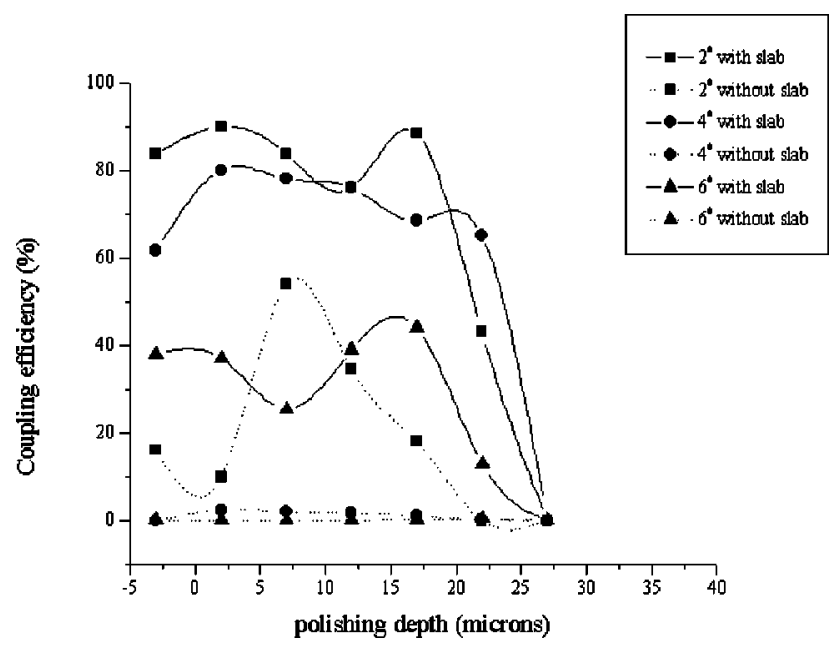

Fig. 9 Coupling efficiency versus polishing depth for various angles. The index-matching slab role is emphasized by the comparison between coupling with (solid line) and without the slab (dotted line). A negative polishing depth means that more than half of the MMF was polished. 


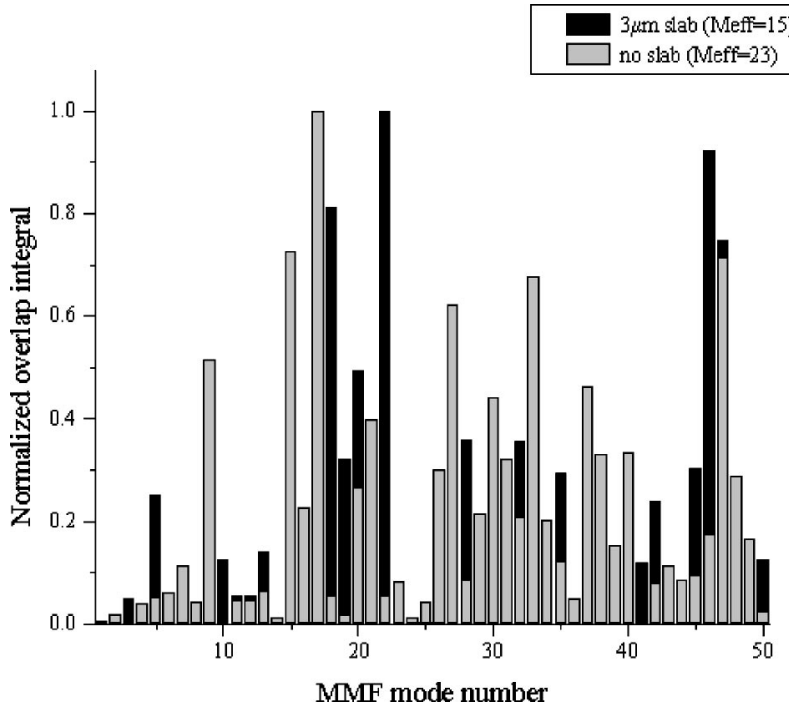

Fig. 10 Comparison of mode excitation with and without the indexmatching slab for coupling angle of $4 \mathrm{deg}$ and polishing depth of 15 $\mu \mathrm{m}$. The intermediate slab provides a more selective coupling illustrated by a smaller $M_{\text {eff }}$.

enables fairly stable efficiency for large polishing depth variations. Simulations also showed a more selective coupling when the slab is present, as shown in Fig. 10. A coupling scheme without an index-matching film excites about $16 \%$ more modes of all orders, increasing the risk of DMD. Only a small variation $( \pm 2 \%)$ of the number of modes excited was observed for a $4-\mu \mathrm{m}$ thickness variation of the slab. The presence of the index-matching film appears therefore to be very useful for efficient coupling and bandwidth enhancement of perturbed MMF since it enables more efficient coupling to fewer modes.

\section{Conclusion}

We presented an analysis of selective mode excitation based on nonaxial evanescent coupling from a SMF to a gradient-index fiber with a perturbed index profile. We showed the coupling properties were improved by the presence of a 3- $\mu \mathrm{m}$ index-matching film.

We used this method to improve the available bandwidth of a perturbed parabolic index profile MMF typically found in LANs. We compared it to other techniques such as OFL and the off-axis technique. The enhancement ratio with respect to the OFL technique is greater than 2 for a large tolerance on polishing depth of MMF and reaches 4.5 for polishing depth of $16 \mu \mathrm{m}$ and coupling angle of $8 \mathrm{deg}$. This technique also proved to excite twice as many modes as the off-axis launch technique (20 to 50\%) for equal or greater bandwidth improvement.

The coupling losses remain below $3 \mathrm{~dB}$ for angles less than $6 \mathrm{deg}$ and large variations in polishing depths. The analysis stresses the role of the index-matching slab as a mode converter for high efficiency and selective coupling.

Because the simulation assumed perfectly smooth surfaces, bandwidth enhancement may decrease if polishing was suboptimal due to the increase number of modes ex- cited. However, the index-matching slab transition is expected to attenuate this effect.

\section{References}

1. D. Cunningham and W. Lane, Gigabit Ethernet Networking, MacMillan, New York (1999).

2. P. F. Checcacci, R. Falciai, and A. M. Sheggi, "Influence of central depression on the dispersion characteristics of a graded index profile," Electron. Lett. 13(13), 378-379 (1977).

3. D. Marcuse, "Calculation of bandwidth from index profiles of optical fibers. 1: theory," Appl. Opt. 18(12), 2073-2080 (1979).

4. L. Jeunhomme and J. P. Pocholle, "Selective mode excitation of graded index optical fibers," Appl. Opt. 17(3), 463-468 (1978).

5. M. Webster, L. Raddatz, I. H. White, and D. G. Cunningham, "A statistical analysis of conditioned launch for gigabit ethernet links using multimode fiber," J. Lightwave Technol. 17(9), 1532-1539 (1999).

6. L. Raddatz, I. H. White, D. G. Cunningham, and M. C. Nowell, "Influence of restricted mode excitation on bandwidth of multimode fiber links," IEEE Photonics Technol. Lett. 10(4), 534-536 (1998).

7. L. Raddatz, I. H. White, D. G. Cunningham, and M. C. Nowell, "An experimental and theoretical study of the offset launch technique for the enhancement of the bandwidth of multimode fiber links," J. Lightwave Technol. 16(3), 324-331 (1998).

8. E. G. Johnson and J. Stack, "Light coupling by a vortex lens into graded index fiber," J. Lightwave Technol. 19(6), 753-759 (2001).

9. S. Tseng and C. Chen, "Side polished fibers," Appl. Opt. 31(18), 3438-3447 (1992).

10. S. Liaw, J. Chiu, and S. Tseng, "Precision side-polished fibers with a long interaction length," Jpn. J. Appl. Phys., Part 1 36(9), 1179-1181 (2000).

11. R. Griffin, J. D. Love, P. R. A. Lyons, D. A. Thorncraft, and S. C. Rasleigh, "Asymmetric multimode couplers," J. Lightwave Technol. 9(11), 1508-1517 (1991)

12. M. B. Miller, "Linear data bus for aircraft application," in Fiber Optic Systems for Mobile Platforms, Proc. SPIE 840, 128-135 (1987).

13. M. S. Whalen and T. H. Wood, "Effectively nonreciprocal evanescent-wave optical-fibre directional coupler," Electron. Lett. 21(5), 176-177 (1985).

14. G. P. Valentin and S. Igor, "Coupling arrangement between a multimode light source and an optical fiber through an intermediate optical fiber," U.S. Patent No. 5999673 (July 1999).

15. A. Cozannet and M. Treheux, "Skew rays in optical fibers," Appl. Opt. 14(6), 1345-1349 (1975).

16. G. R. Hadley, "Full-vector waveguide modeling using an iterative finite-difference method with transparent boundary conditions," $J$ Lightwave Technol. 13(3), 465-469 (1993).

17. S. Jungling and J. C. Chen, "A study and optimization of eigenmode calculations using the imaginary distance beam-propagation method," IEEE J. Quantum Electron. 30(9), 2098-2105 (1994).

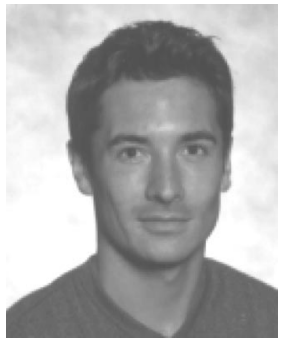

Laurent Vaissié received his MSc degree in engineering physics from Ecole Nationale Supérieure de Physique, Marseille, in 1998, an advanced study degree in geophysics from the University of Pau and Montpellier in 1999 for modeling wavefront propagation in 3-D complex structures using caustics, and his MSc degree in optics in 2001 from the School of Optics/CREOL where he is currently working toward his $\mathrm{PhD}$ degree in the field of integrated optics. His current research interests include simulation and integration of diffractive elements on side-polished fibers and laser diodes.

Eric G. Johnson received his BSc degree in physics from Purdue University in 1985, his MSc degree in electrical engineering from the University of Central Florida in 1989 and his PhD degree in electrical engineering from the University of Alabama, Huntsville, in 1996. Since 2000 he has been an assistant professor of optical engineering with the School of Optics/CREOL. Prior to joining CREOL, he was the vice president of research \& development with Digital Optics Corporation in Charlotte, North Corollina. He has also held positions at SY Technology, Teledyne Brown Engineering, and Martin Marietta. His research interests include diffractive and microphotonic systems, data communications, and nanophotonics. He is a member of OSA and IEEE. 